\title{
Primary tumor location is an important predictor of survival in pulmonary adenocarcinoma
}

This article was published in the following Dove Medical Press journal: Cancer Management and Research

\section{Zhe Wang ${ }^{1,2}$ \\ Minghuan $\mathrm{Li}^{2}$ \\ Feifei Teng ${ }^{2}$ \\ Li Kong ${ }^{2}$ \\ Jinming $\mathrm{Yu}^{2}$}

'School of Medicine, Shandong University, Jinan, Shandong, China; ${ }^{2}$ Department of Radiation Oncology, Shandong Cancer Hospital Affiliated to Shandong University, Jinan, Shandong, China
Correspondence: Jinming Yu; Minghuan Li

Department of Radiation Oncology, Shandong Cancer Hospital Affiliated to Shandong University, Jiyan Road 440, Jinan, Shandong 250I I7, China

Tel +8653167626971

Fax +8653187984079

Email sdyujinming@163.com; sy_Imh200I@I63.com
Purpose: The prognostic value of tumor location in pulmonary adenocarcinoma (ADC) is controversial. We compared the prognosis and relevant data between central-type ADC (CT-ADC) and peripheral-type ADC (PT-ADC) in order to identify the reasons for the different outcomes between them and to improve the treatment strategy and prognosis of these two types.

Patients and methods: Data of 256 patients with pathologically diagnosed ADC were retrospectively reviewed. The prognostic factors for disease-free survival (DFS), progression-free survival (PFS), and overall survival (OS) were analyzed using univariate and multivariate analyses. Results: A total of 124 and 132 patients had CT-ADC and PT-ADC, respectively. CT-ADC was associated with an earlier age, poorer Karnofsky Performance Status (KPS), higher rates of advanced stage, bone metastasis, contralateral pulmonary metastasis, and pleural effusion. Besides, CT-ADC showed a trend toward lower rate of EGFR mutation. Patients with CT-ADC had a significantly shorter PFS/DFS and OS than did those with PT-ADC. Multivariate analysis revealed that advanced stage, central-type location, EGFR wild-type, no surgery, presence of COPD, and interstitial lung disease (ILD) were independent poor prognostic factors for OS. The rate of surgery was significantly lower in patients with CT-ADC. Among patients with ILD or COPD, OS is shorter in patients with central- than peripheral-type tumors.

Conclusion: CT-ADC is associated with poorer survival than PT-ADC and the lower rate of surgery in patients with CT-ADC is an important reason for this. Tumor location of pulmonary ADC plays a critical role in predicting prognosis and choosing therapeutic strategies.

Keywords: lung cancer, EGFR, surgery, clinical stage, comorbidity

\section{Introduction}

Non-small-cell lung cancer (NSCLC) accounts for $>80 \%$ of all lung cancers. Pulmonary adenocarcinoma (ADC) and squamous cell carcinoma (SQCC) are the most frequent histological subtypes, accounting for $40 \%-60 \%$ and $30 \%$ of NSCLC cases, respectively. ${ }^{1-3}$ Furthermore, the proportion of ADC has been rising dramatically in the past few years because of decreased smoking behavior. ${ }^{2-4}$ For early-stage ADC, surgical resection is preferred. And for advanced-stage or inoperable ADC, chemotherapy with or without radiation therapy is the main therapeutic strategy. Besides, with the development of molecular target therapy, patients with EGFR mutations can benefit more from tyrosine kinase inhibitors.

Lung cancer can also be subgrouped into central and peripheral types according to the location of the primary tumor. ADC has long been believed to be peripheraltype (PT-ADC) mainly. However, recent studies have found that the incidence rate of 
central-type ADC (CT-ADC) has been increasing, and the prognostic value of tumor location in ADC is controversial. ${ }^{5-8}$ The majority of these studies supported the conclusion that central location was associated with a high incidence of lymph node involvement and a poor prognosis..$^{5-7}$ But another study reported a better clinical outcome in patients with CT-ADC. ${ }^{8}$ However, no information regarding EGFR status and treatment is available, though the therapeutic strategy is an important prognostic factor. On the other hand, as two common comorbidities of lung cancer, COPD and interstitial lung disease (ILD) are associated with oncogenesis and shorter survival in patients with NSCLC. ${ }^{9-15}$ Besides, the prevalence of ILD is lower in central- than in peripheraltype lung cancer, ${ }^{16-18}$ whereas the prevalence of COPD is higher in central-type lung cancer. ${ }^{19}$ But SQCC is the most frequent histological type in patients with ILD or COPD. ${ }^{10,16}$ Whether the incidences of these comorbidities differ between CT-ADC and PT-ADC and their impacts on survival have remained unclear.

The prognostic value of tumor location in ADC is controversial. And the factors leading to different prognosis between CT-ADC and PT-ADC are unclear. So in the present study, we compared the clinical characteristics, laboratory data, EGFR status, incidences of ILD and COPD, treatment strategy, response and survival between CT-ADC and PT-ADC in order to identify the reasons for the different outcomes between them and to improve the treatment strategy and prognosis of these two types.

\section{Patients and methods}

\section{Patients}

This study was approved by the Institutional Review Board of Shandong Cancer Hospital and conformed to Declaration of Helsinki. The number of approval document was 20161005. All patients provided informed consent. The patient consent was written informed consent. The study outcomes would not affect the future management of the patients. We retrospectively reviewed the medical records of all patients with pathologically confirmed primary pulmonary ADC who presented to Shandong Cancer Hospital from July 2014 to September 2016. All patients had a computed tomographic scan of the chest, fiberoptic bronchoscopy examination, spirometry test, and EGFR status test before receiving any treatment. The relevant clinical and laboratory data were collected from their medical records. The median follow-up period was 23.9 months (95\% CI: 22.0 -25.8 months).

\section{Definition of tumor location}

Based on previous studies, ${ }^{5,20,21}$ primary tumors involving segmental and/or lobar bronchus were considered to be central and any tumor not meeting this criterion was considered to be peripheral. The location of the primary tumor was defined by fiberoptic bronchoscopy.

\section{Diagnosis of COPD and ILD}

The diagnosis of COPD was based on Global Strategy for the Diagnosis, Management, and Prevention of Chronic Obstructive Pulmonary Disease. ${ }^{22}$ A post-bronchodilator FEV1/ FVC $<0.70$ confirmed the diagnosis of COPD in patients with predisposing risks and appropriate symptoms, such as dyspnoea, chronic cough, or sputum production.

According to the American Thoracic Society/European Respiratory Society/Japanese Respiratory Society/Latin American Thoracic Association statement, ${ }^{23}$ ILD was classified into ILD with a usual interstitial pneumonia (UIP) pattern, ILD with a possible UIP pattern, and ILD inconsistent with a UIP pattern. ILD with a UIP pattern was defined as having all of the following characteristics: subpleural and basal predominance, reticular abnormality, and honeycombing with or without traction bronchiectasis. The case was classified as a possible UIP pattern if the patient had the above characteristics except honeycombing.

\section{Statistical analysis}

Statistical analysis was performed using SPSS software (version 22.0). We analyzed the distribution of continuous data by Kolmogorov-Smirnov test and found that they were all non-normally distributed. The continuous data were reported as median and range and compared between patients by Mann-Whitney $U$ test in our study. Comparison of proportions of the categorical data was analyzed by $\chi 2$ or Fisher's exact test if appropriate. Treatment response was determined by RECIST 1.1 criteria. Disease-free survival (DFS) was defined as the time between the date of tumor resection and the date of first known local or distant recurrence. Progression-free survival (PFS) was defined as the time between the start of active treatment except surgery and date of disease progression or death. Overall survival (OS) was defined as the time between the date of diagnosis and date of death from any cause. PFS and OS curves were constructed by the Kaplan-Meier method. Differences in survival were compared using the log-rank test for univariate analysis. And Cox-proportional hazards regression (forward likelihood ratio model) was performed for multivariate analysis. A two-sided $P<0.05$ was considered statistically significant. 


\section{Results}

\section{Patient characteristics}

There were 256 patients with pathologically confirmed pulmonary ADC included in our study. A total of 124 patients
(48.4\%) had central-type tumor and 132 patients (51.6\%) had peripheral-type tumor. Relevant clinical and laboratory characteristics are presented in Table 1. CT-ADC was associated with an earlier age $(P=0.001)$ and poorer Karnofsky

Table I Relevant clinical and laboratory features

\begin{tabular}{|c|c|c|c|c|}
\hline & All, $\mathbf{N}=\mathbf{2 5 6}$ & $\begin{array}{l}\text { Central, } \\
N=\mid 24 \text { (48.4\%) }\end{array}$ & $\begin{array}{l}\text { Peripheral, } \\
N=\mid 32 \text { (5 I.6\%) }\end{array}$ & $P$-value \\
\hline Age, years (range) & $58(32-77)$ & $55(32-77)$ & $60(37-77)$ & 0.001 \\
\hline Gender & & & & 0.28 \\
\hline Male & $108(42.2 \%)$ & 48 (38.7\%) & 60 (45.5\%) & \\
\hline Female & I 48 (57.8\%) & 76 (61.3\%) & 72 (54.5\%) & \\
\hline KPS & $90(60-100)$ & $90(70-95)$ & $90(60-100)$ & 0.002 \\
\hline \multicolumn{5}{|l|}{ Smoking status } \\
\hline Never & 179 (69.9\%) & 92 (74.2\%) & 87 (65.9\%) & 0.15 \\
\hline Ever & 77 (30.1\%) & $32(25.8 \%)$ & $45(34.1 \%)$ & \\
\hline Cigarette index & $600(200-2000)$ & $600(400-1200)$ & $600(200-2000)$ & 0.69 \\
\hline Clinical stage & & & & $<0.001$ \\
\hline I & 40 (15.6\%) & $8(6.5 \%)$ & 32 (24.2\%) & \\
\hline II & $7(2.7 \%)$ & $6(4.8 \%)$ & $\mathrm{I}(0.8 \%)$ & \\
\hline IIIA & $56(21.9 \%)$ & $24(19.3 \%)$ & $32(24.2 \%)$ & \\
\hline$I I I B+I I I C$ & $15(5.9 \%)$ & $10(8.1 \%)$ & $5(3.8 \%)$ & \\
\hline IV & $138(53.9 \%)$ & $76(61.3 \%)$ & $62(47.0 \%)$ & \\
\hline \multicolumn{5}{|l|}{ Distal metastasis } \\
\hline Brain & $53(20.7 \%)$ & 31 (39.1\%) & $22(16.7 \%)$ & 0.10 \\
\hline Bone & $68(26.6 \%)$ & $44(35.5 \%)$ & $24(18.2 \%)$ & 0.002 \\
\hline Liver & $28(10.9 \%)$ & $16(12.9 \%)$ & $12(9.1 \%)$ & 0.33 \\
\hline Others & $36(14.1 \%)$ & $16(12.9 \%)$ & $20(15.2 \%)$ & 0.61 \\
\hline \multicolumn{5}{|l|}{ EGFR status } \\
\hline Wild-type & 124 (48.4\%) & 68 (54.8\%) & 56 (42.4\%) & 0.06 \\
\hline Mutant-type & $132(51.6 \%)$ & $56(45.2 \%)$ & $76(57.6 \%)$ & \\
\hline 2I L858R & $75(56.8 \%)$ & $28(50.0 \%)$ & 47 (61.9\%) & 0.14 \\
\hline $19 \mathrm{del}$ & 52 (39.4\%) & 24 (42.9\%) & $28(36.8 \%)$ & \\
\hline Others & $5(3.8 \%)$ & $4(7.1 \%)$ & $\mathrm{I}(\mathrm{I} .3 \%)$ & \\
\hline $\operatorname{CEA}(n g / m L), n=243$ & $6.70(0.50-709.30)$ & $8.11(0.50-595.20)$ & $5.12(0.89-709.30)$ & 0.07 \\
\hline $\mathrm{LDH}(\mathrm{U} / \mathrm{L}), \mathrm{n}=243$ & $188(107-1996)$ & $191(107-1996)$ & $186(111-477)$ & 0.07 \\
\hline LMR, $n=254$ & $3.15(0.77-13.71)$ & $2.97(|.3|-|3.7|)$ & $3.64(0.77-8.58)$ & 0.006 \\
\hline Contralateral pulmonary metastasis & & & & 0.001 \\
\hline No & 185 (72.3\%) & 78 (62.9\%) & $107(81.1 \%)$ & \\
\hline Yes & $71(27.7 \%)$ & $46(37.1 \%)$ & $25(18.9 \%)$ & \\
\hline Visceral pleural nodule & & & & $0.7 \mathrm{I}$ \\
\hline No & $158(61.7 \%)$ & $78(62.9 \%)$ & $80(60.6 \%)$ & \\
\hline Yes & $98(38.3 \%)$ & $46(37.1 \%)$ & $52(39.4 \%)$ & \\
\hline Interlobar pleural nodule & & & & 0.10 \\
\hline No & $20 \mathrm{I}(78.5 \%)$ & 92 (74.2\%) & $109(82.6 \%)$ & \\
\hline Yes & $55(21.5 \%)$ & $32(25.8 \%)$ & $23(17.4 \%)$ & \\
\hline Pleural effusion & & & & $<0.001$ \\
\hline No & $187(73.0 \%)$ & 69 (55.6\%) & I I 8 (89.4\%) & \\
\hline Yes & 69 (27.0\%) & 55 (44.4\%) & $14(10.6 \%)$ & \\
\hline COPD & & & & 0.53 \\
\hline No & 228 (89.1\%) & I I 2 (90.3\%) & I I 6 (87.9\%) & \\
\hline Yes & $28(10.9 \%)$ & $12(9.7 \%)$ & $16(12.1 \%)$ & \\
\hline ILD & & & & 0.76 \\
\hline No & I 88 (73.4\%) & 90 (72.6\%) & 98 (74.2\%) & \\
\hline Yes & $68(26.6 \%)$ & $34(27.4 \%)$ & 34 (25.8\%) & \\
\hline
\end{tabular}

Abbreviations: CEA, carcino-embryonic antigen; ILD, interstitial lung disease; LDH, lactate dehydrogenase; LMR, lymphocyte-monocyte ratio; KPS, Karnofsky Performance Status. 
Performance Status (KPS) $(P=0.002)$. More importantly, central-type patients presented with higher rates of advanced stage $(P<0.001)$, bone metastasis $(P=0.002)$, contralateral pulmonary metastasis $(P=0.001)$, pleural effusion $(P<0.001)$, and lower lymphocyte-monocyte ratio (LMR) $(P=0.006)$. CTADC showed a trend toward lower rate of EGFR mutation compared to PT-ADC, though the difference did not reach statistical significance $(P=0.06)$. It indicated that CT-ADC presented with more malignant clinical characteristics.

\section{Treatment with and response of CT-ADC and PT-ADC}

All patients included in our study received active treatment (Table 2). The rate of surgery was significantly higher in patients with PT-ADC than CT-ADC $(53.0 \%$ and $21.0 \%$, respectively, $P<0.001)$. Limited to patients with operable stages (I-IIIA), the surgical rate was also higher in PTADC patients $(P=0.002)$. The rates of patients receiving chemoradiotherapy and target therapy were similar between patients with CT-ADC and PT-ADC. Also, the response rate to chemotherapy with or without radiotherapy was similar between patients with CT-ADC and peripheral-type ADC ( $49.3 \%$ and $40.0 \%$ respectively, $P=0.31$ ).

\section{Prognosis of CT-ADC and PT-ADC}

The 2-year PFS rate of all patients was $29.4 \%$ (95\% CI: $23.1 \%-35.7 \%)$ and the 2-year OS rate was $62.0 \%(95 \%$ CI: $55.7 \%-68.3 \%$ ). The 2-year PFS rates of CT-ADC and PT-ADC patients were $17.6 \%$ (95\% CI: $10.0 \%-25.2 \%)$ and 41.8\% (95\% CI: 32.6\%-51.0\%), respectively. And the 2-year OS rates of CT-ADC and PT-ADC patients were 49.1\% (95\% CI: $39.3 \%-58.9 \%$ ) and $73.0 \%$ (95\% CI: $65.2 \%-80.8 \%)$, respectively. Univariate analysis showed that a poor KPS $(P=0.005)$, advanced stage $(P<0.001)$, central-type location $(P<0.001)$, EGFR wild-type $(P<0.001)$, low LMR $(P=0.04)$, no surgery $(P<0.001)$, presence of contralateral pulmonary metastasis $(P=0.04)$, interlobar pleural metastasis $(P=0.008)$, pleural effusion $(P<0.001)$, COPD $(P=0.02)$, and ILD $(P<0.001)$ were associated with poor OS (Table 3). Multivariate analysis revealed that advanced stage (HR $=2.14[1.01-4.51], P=0.046)$, central-type location $(\mathrm{HR}=2.14$ [1.01-4.51], $P=0.002)$, EGFR wild-type $(\mathrm{HR}=0.20[0.12-$ $0.32], P<0.001)$, no surgery $(\mathrm{HR}=0.36[0.16-0.79], P=0.01)$, presence of COPD $(\mathrm{HR}=1.83$ [1.00-3.32], $P=0.048)$, and ILD $(\mathrm{HR}=2.43[1.55-3.81], P<0.001)$ were independent poor prognostic factors for OS (Table 3 ). Besides, advanced stage $(\mathrm{HR}=2.80$ [1.92-4.80], $P<0.001)$, central-type location $(\mathrm{HR}=0.67$ [0.48-0.94], $P=0.02)$, EGFR wild-type $(\mathrm{HR}$ $=0.71[0.51-1.00], P=0.049)$, and presence of ILD $(\mathrm{HR}=1.87$ [1.29-2.70], $P=0.001)$ were independent poor prognostic indicators for PFS/DFS (Table 3). The OS curves of patients with central- and peripheral-type tumors are presented in Figure 1A $(P<0.001)$.

To clarify the predictive effects of tumor location and stage, we classified all patients into four groups as CT-ADC and early stage (stages I-III); CT-ADC and advanced stage (stage IV); PT-ADC and early stage (stages I-III); and PTADC and advanced stage (stage IV). Patients with PT-ADC and early stages had best prognosis, and patients with CT$\mathrm{ADC}$ and advanced stages showed the poorest prognosis (Figure 1B, C; median PFS/DFS, 890 and 225 days, respectively, $P<0.001$; median OS, not reached [NR] and 518 days, respectively, $P<0.001)$. However, the prognosis of patients with CT-ADC and early stages was not significantly different

Table 2 Treatment and response to first-line chemotherapy with or without radiotherapy

\begin{tabular}{|c|c|c|c|c|}
\hline & All & Central & Peripheral & $P$-value \\
\hline First-line treatment & & & & \\
\hline Surgery & 96 & 26 & 70 & $<0.001$ \\
\hline Surgery (I-IIIA) & 82 & 24 & 58 & 0.002 \\
\hline Chemotherapy & 96 & 60 & 36 & $<0.001$ \\
\hline Chemoradiotherapy & 23 & 9 & 14 & 0.35 \\
\hline Neoadjuvant chemotherapy & 16 & 14 & 2 & 0.001 \\
\hline EGFR-TKI & 25 & 15 & 10 & 0.22 \\
\hline Response to chemotherapy with or without radiotherapy & $\mathrm{N}=119$ & $\mathrm{~N}=69$ & $\mathrm{~N}=50$ & \\
\hline$C R+P R$ & 54 & 34 & 20 & \\
\hline SD & 46 & 26 & 20 & \\
\hline PD & 19 & 9 & 10 & \\
\hline Objective response rate & $45.4 \%$ & $49.3 \%$ & $40.0 \%$ & 0.31 \\
\hline Disease control rate & $84.0 \%$ & $87.0 \%$ & $80.0 \%$ & 0.31 \\
\hline
\end{tabular}

Abbreviations: CR, complete response; PD, progressive disease; PR, partial response; SD, stable disease; TKI, tyrosine kinase inhibitor. 


\begin{tabular}{|c|c|c|c|c|c|c|}
\hline 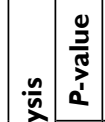 & ָ̃ & $\begin{array}{l}\text { tơ } \\
0\end{array}$ & ธ్ & $\begin{array}{l}\bar{o} \\
\dot{v}\end{array}$ & 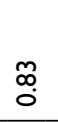 & $\bar{o}$ \\
\hline 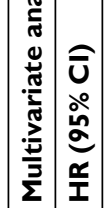 & 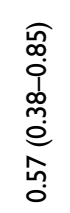 & 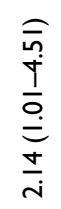 & 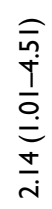 & 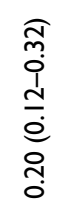 & $\begin{array}{l}\widehat{o} \\
\bar{j} \\
\frac{j}{0} \\
o \\
\circ \\
\stackrel{0}{0}\end{array}$ & $\begin{array}{l}\text { న } \\
0 \\
1 \\
1 \\
0 \\
0 \\
\text { m. }\end{array}$ \\
\hline
\end{tabular}

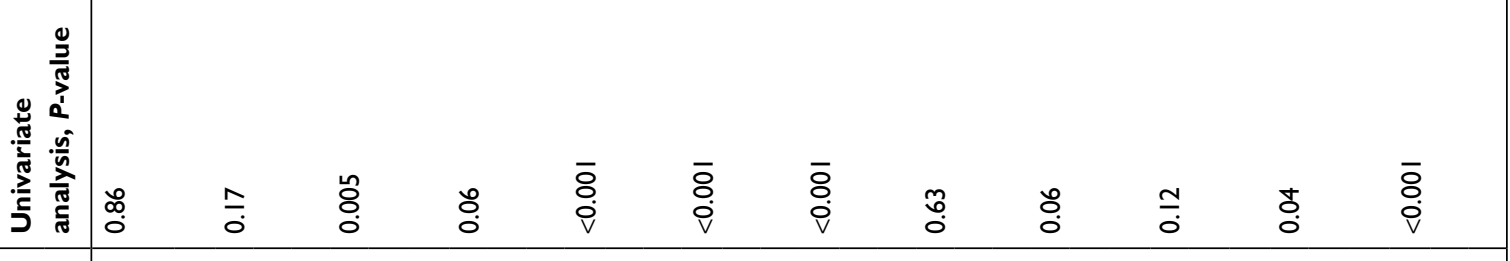

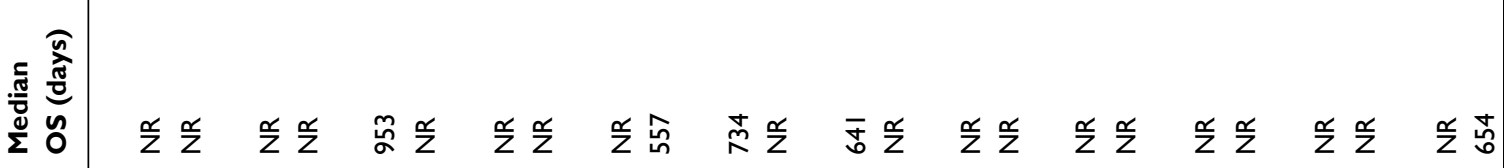

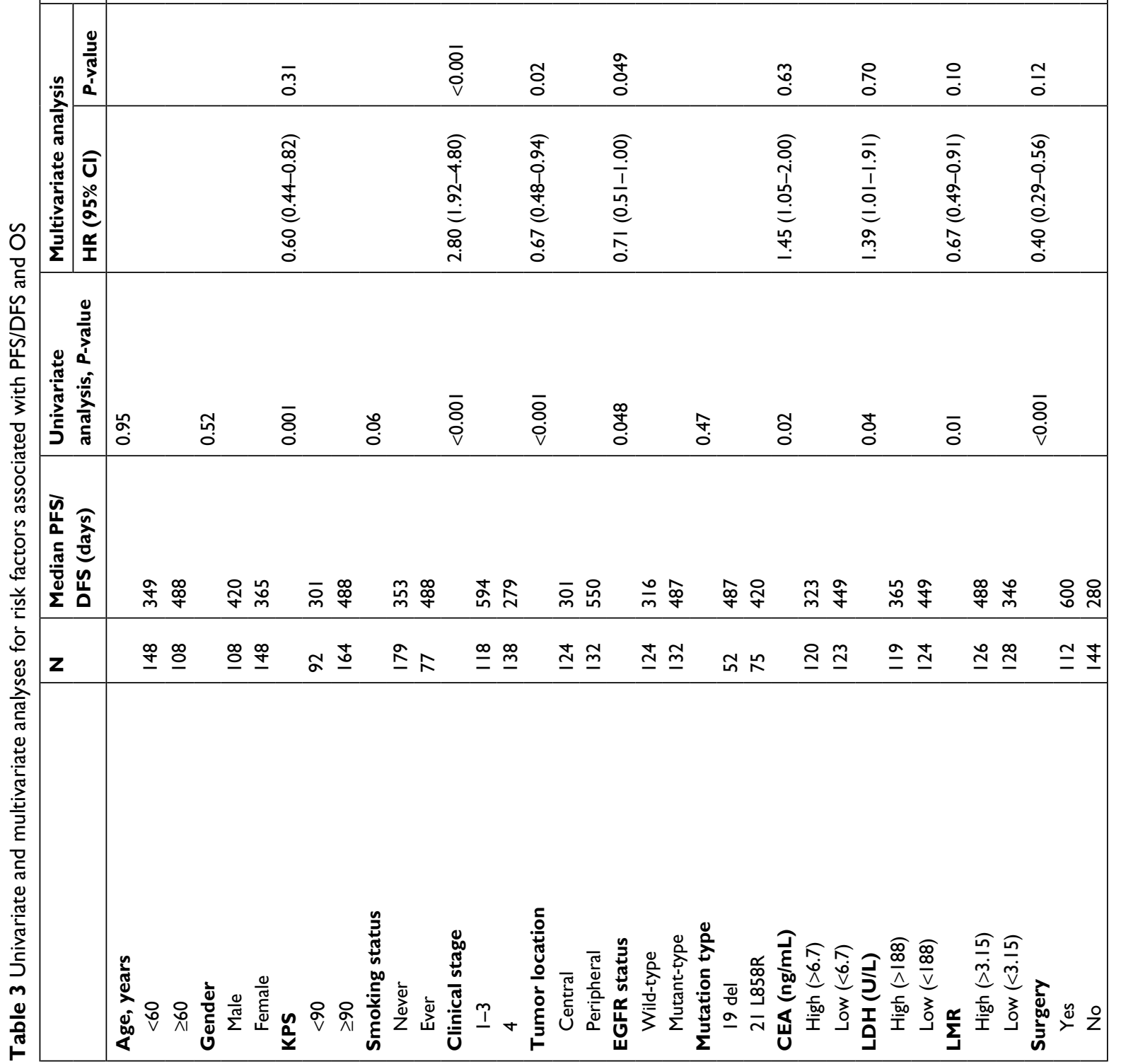




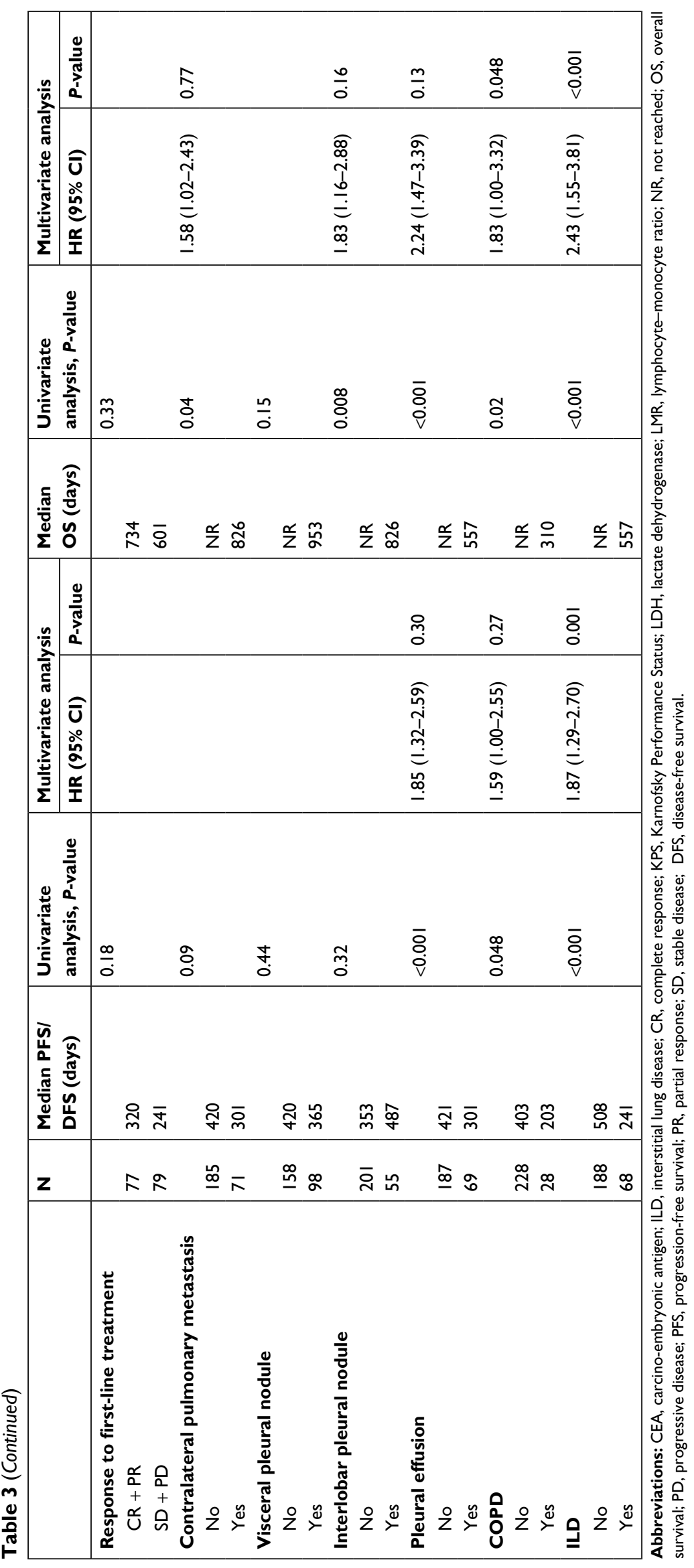



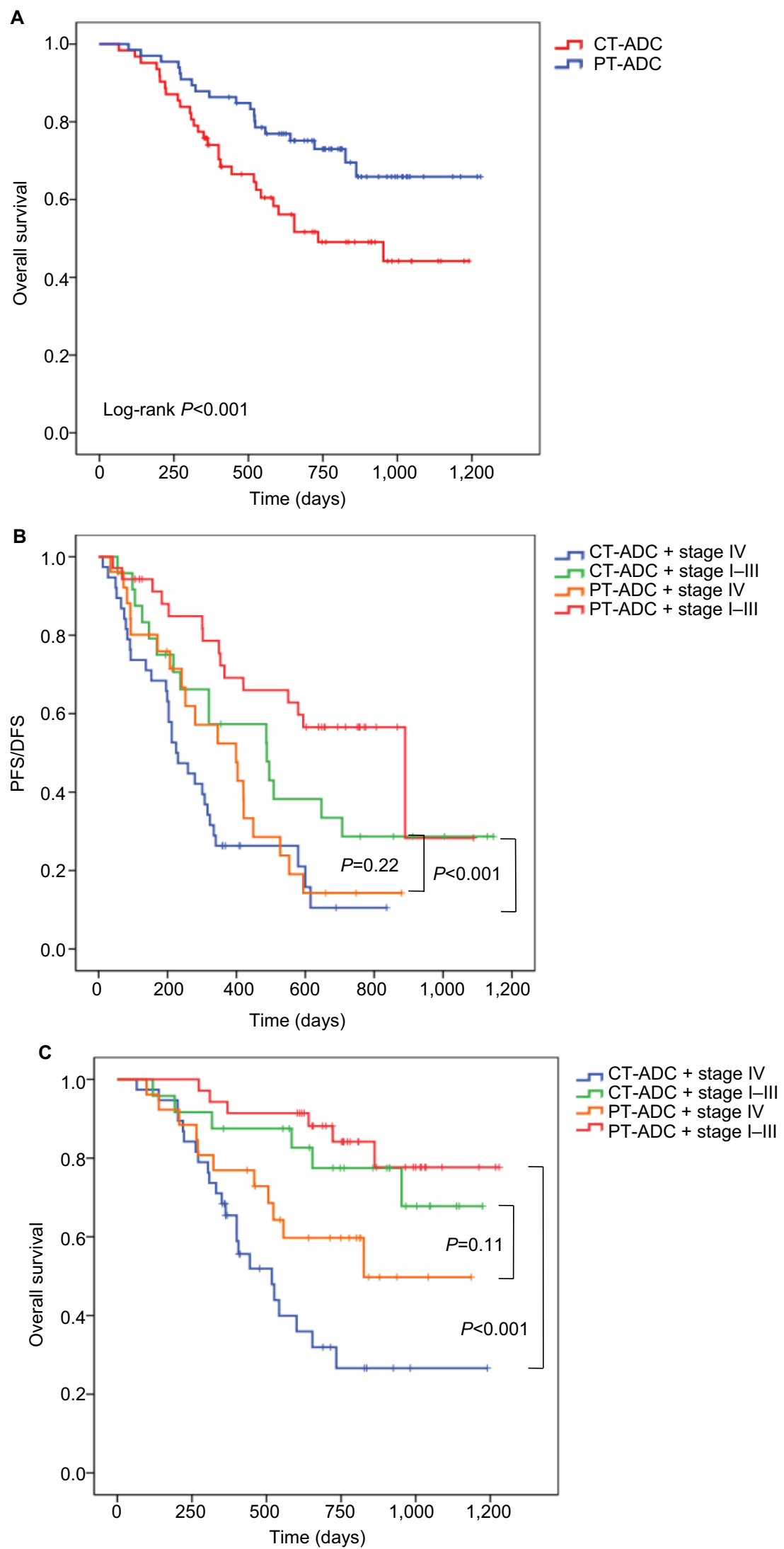

Figure I Kaplan-Meier curves of (A) overall survival in patients with pulmonary adenocarcinoma according to tumor location, (B) PFS/DFS in patients with pulmonary adenocarcinoma according to tumor location and clinical stage, and $(\mathbf{C})$ overall survival in patients with pulmonary adenocarcinoma according to tumor location and clinical stage.

Abbreviations: DFS, disease-free survival, PFS, progression-free survival; CT-ADC, central-type ADC; PT-ADC, peripheral-type ADC. 
from those with PT-ADC and advanced stages (Figure 1B, C; median PFS/DFS, 488 and 399 days, respectively, $P=0.22$; median OS, NR and 826 days, respectively, $P=0.11$ ). This indicated that the prognostic value of tumor location was as critical as that of clinical stage.

To clarify the effect of surgery in patients' outcomes with CT-ADC and PT-ADC, we analyzed OS in patients who underwent surgery and who did not. Limited to patients who did not have surgical resections, the superiority of OS and PFS for patients with PT-ADC disappeared (OS: Figure 2A, $P=0.23$; PFS: Figure $2 \mathrm{~B}, P=0.76)$. Similarly, limited to patients who underwent surgery, there was no survival difference between patients with central- and peripheral-type tumors (Figure 2C, $P=0.26$ ), whereas the difference in DFS still remained (Figure 2D; median DFS, 487 and 890 days, respectively, $P=0.01$ ). This indicated that surgery was an important reason for the shorter survival of patients with CT-ADC.

\section{Survival of patients with ILD or COPD}

Although the presence of ILD or COPD did not significantly differ between patients with CT-ADC and PT-ADC (Table 1), they were the independent prognostic factors for poor survival (Table 3). Furthermore, coexistence of ILD and COPD was associated with even shorter OS (Figure 3A; median, NR, 601 and 203 days in COPD only, ILD only, and coexistence of ILD and COPD, respectively, $P<0.05)$. In patients without ILD, the survival of central-type patients did not significantly differ from that of peripheral-type patients (Figure 3B; $P=0.17)$. However, in patients with ILD, OS was much longer in patients with peripheral- than central-type tumors (Figure $3 \mathrm{C} ; P=0.01)$. Similar results can be drawn in the patients with or without COPD (Figure 3D, E).

\section{Discussion}

The present study revealed that the incidence of CT-ADC was comparable with that of PT-ADC. More importantly, we demonstrated that patients with CT-ADC presented with higher rates of advanced stage and more malignant clinical characteristics. And central location was an independent prognostic factor for poor survival. Besides, the rate of surgical resection was lower in patients with CT-ADC, which was one of the reasons for their shorter survival. Lastly, though
A.

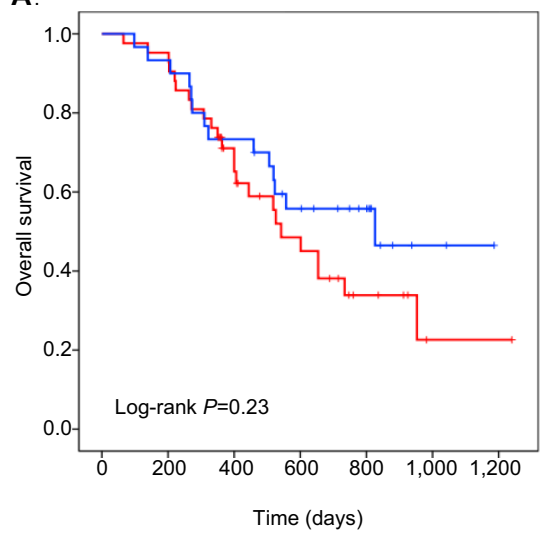

C

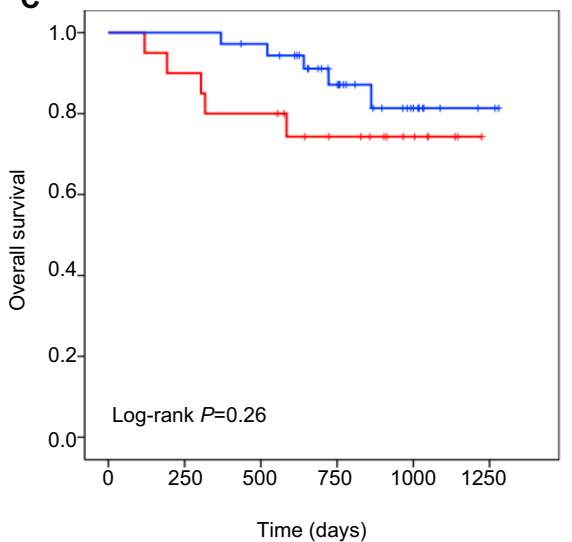

$\rightarrow$ CT-ADC $\neg$ PT-ADC

B

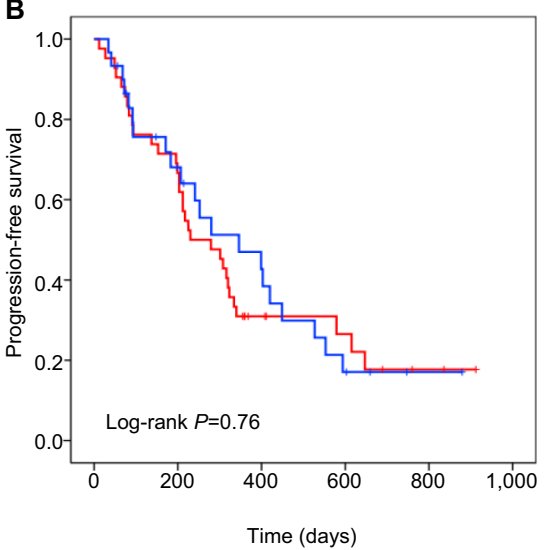
$\underset{\sim \mathrm{PT}-\mathrm{ADC}}{\mathrm{C} T-A D C}$

D
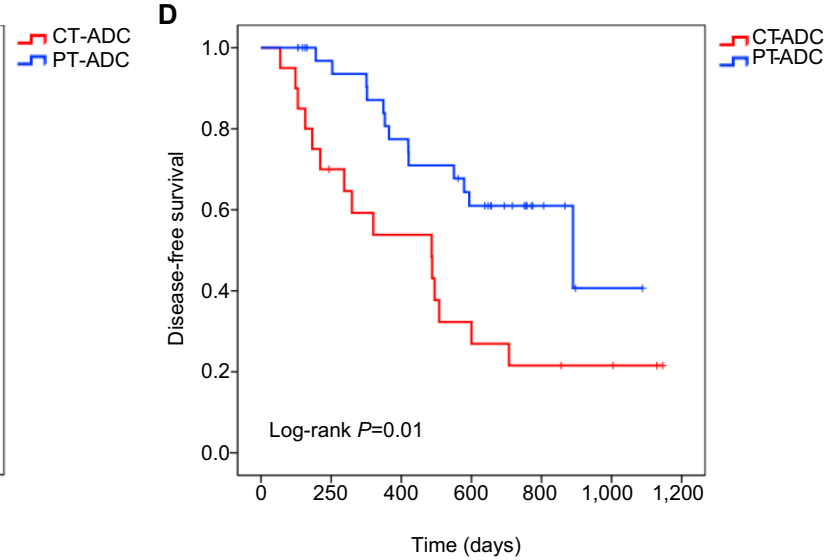

Figure 2 Kaplan-Meier curves of (A) overall survival in patients without surgery according to tumor location, (B) progression-free survival in patients without surgery according to tumor location, (C) overall survival in patients with surgery according to tumor location, and (D) disease-free survival in patients with surgery according to tumor location.

Abbreviations: CT-ADC, central-type ADC; PT-ADC, peripheral-type ADC. 

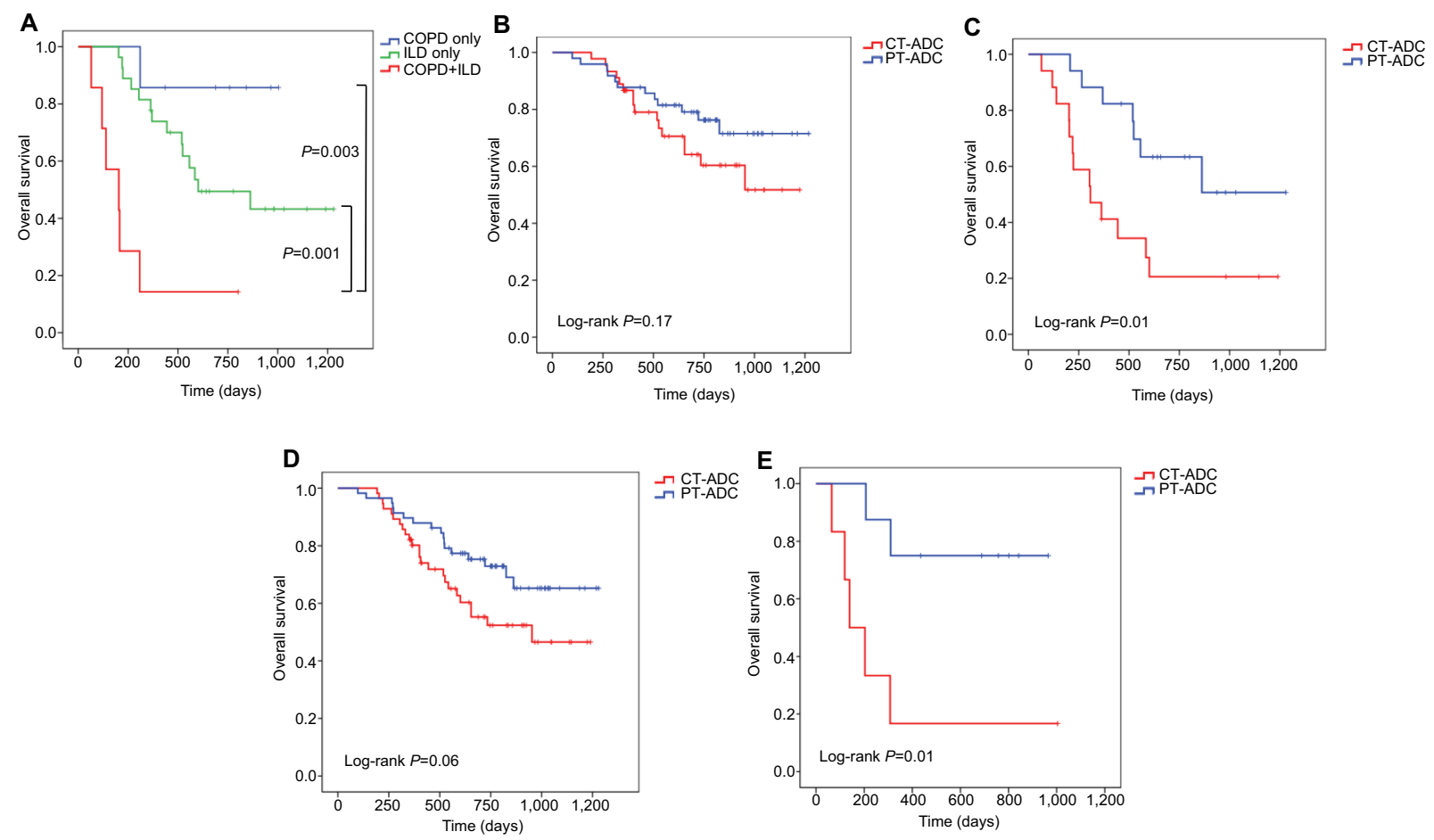

Figure 3 Kaplan-Meier curves of overall survival in patients (A) with pulmonary adenocarcinoma according to comorbidities, (B) without ILD according to tumor location, (C) with ILD according to tumor location, (D) without COPD according to tumor location, and (E) with COPD according to tumor location.

Abbreviations: ILD, interstitial lung disease; CT-ADC, central-type ADC; PT-ADC, peripheral-type ADC.

the incidence rates of COPD or ILD were similar between patients with CT-ADC and PT-ADC, OS was shorter in patients with central- than peripheral-type tumors in patients with comorbidities. Our study suggests that elevated rate of surgery might improve the survival of CT-ADC. And patients with coexisting CT-ADC and ILD/COPD showed poorer prognosis and might need more care.

Few studies have investigated the prognostic value of primary tumor location in patients with $\mathrm{ADC}^{5-8}$ but their results are controversial. The study by Ito et $\mathrm{al}^{8}$ revealed that central location was an indicator for better survival in pN2 NSCLC cases. But other studies showed that CT-ADC was associated with more lymph node involvement, advanced stage, and a poor prognosis, ${ }^{5-7}$ which was consistent with the results of our study. Different inclusion criterion and definition of tumor location might be the reasons for such contrasting results. In our study, patients with CT-ADC presented with more malignant clinical characteristics, such as bone metastasis, contralateral pulmonary metastasis, pleural effusion, and lower lymphocytemonocyte ratio. Furthermore, our study revealed that the central location was a stage-independent prognostic factor for patients with ADC. And the survival of patients with CT-ADC and early stages was not significantly different from those with PT-ADC and advanced stages, which indicates that the prognostic value of tumor location is as important as clinical stage.

Most importantly, the present study is the first to compare the treatment with and treatment response between CT-ADC and PT-ADC. Our study showed that surgical resection was more frequently chosen for PT-ADC than CT-ADC. And when we classified patients according to therapeutic strategies, the superiority of OS for patients with PT-ADC disappeared both in surgery and in non-surgery groups. Together with the result that surgery was an independent prognostic factor for better survival, the low rate of surgery in patients with CT-ADC is a critical reason for their poor prognosis. There are two potential reasons for this phenomenon. First, patients with CT-ADC presented with higher rates of advanced stage, bone metastasis, contralateral pulmonary metastasis, and pleural effusion, which limited the utilization of surgery. Second, the operation difficulty and risk increase with central location since the tumors usually abut primary bronchus, great vessels, or pericardium. Although no information has been provided about surgical resection techniques in the current study, a previous report ${ }^{24}$ revealed that there were more patients with central NSCLC in the open surgery group than in the video-assisted thoracic surgery group 
(24.1\% vs $4.5 \%)$. But with the improvement of thoracoscopic bronchial sleeve resection, ${ }^{25,26}$ the rate of surgery in patients with central-type tumors might increase in the future so as to prolong their survival. In accordance with the study by Sun et al, ${ }^{5}$ DFS was shorter in patients with CT-ADC in our study. And this indicates that tumor location should be considered as an important factor for choosing adjuvant chemotherapy or radiotherapy. As another significant prognostic factor, the rate of advanced stage was higher in central-type patients. Although the reason for this is unclear, peripheral tumors might be more easily discovered by routine medical examination techniques, whereas central-type tumors may often be diagnosed at a late stage and accompanied with lymph node metastases. ${ }^{5,6,21}$ Another possibility is that peripheraltype tumors generally have a more slow growth pattern. A relatively longer period might be permitted for detection of PT-ADC at an early stage. ${ }^{21}$

ILD and COPD are two common comorbidities of lung cancer. Their relationships with oncogenesis and shorter survival in patients with NSCLC are well known. ${ }^{9-15}$ However, evidence on the comparison of comorbidities between CT-ADC and PT-ADC is lacking. In the current study, we compared the incidences of ILD/COPD and evaluated their impacts on long-term survival of patients with CT-ADC and PT-ADC. But unlike previous studies, ${ }^{16-19}$ the incidences of ILD/COPD did not significantly differ between patients with CT-ADC and PT-ADC. The reason for this discrepancy is not certain, but there is a possibility that our study focused only on pulmonary ADC, whereas the previous studies included all histological types of lung cancer, and SQCC was the most common one. The study by Lim et $\mathrm{al}^{19}$ revealed that SQCC accounted for $50.0 \%$ in patients with COPD, whereas ADC accounted for $69.1 \%$ in patients without COPD. Another study $^{13}$ associated with ILD revealed that $46.3 \%$ SQCC patients were presented with ILD but the proportion in ADC patients was only $14 \%$. Besides, smoking behavior is strongly associated with the incidences of ILD and $\mathrm{COPD}^{27,28}$ as well as the distribution of histological types of lung cancer. ${ }^{29}$ In our study, only $30 \%$ patients with ADC were smokers. So the relatively low smoking rate is another possibility for such discrepancy. Despite the fact that the incidences of ILD/COPD did not significantly differ between patients with CT-ADC and PT-ADC, both of them were independent prognostic factors for poor survival, which was also reported in previous studies. ${ }^{9-15}$ It is interesting to note that in patients with ILD or COPD, OS was much longer in patients with peripheral- than central-type tumors, though such difference was not seen in patients without comorbidities. These results suggest that compared to patients with PT-ADC, patients with CT-ADC may be affected more from ILD or COPD and need more active treatment for these comorbidities to improve the prognosis. The chronic airflow limitation that characterizes COPD is caused by a mixture of small airway disease and parenchymal destruction. ${ }^{22}$ Meanwhile, ILD is defined as a specific form of chronic, progressive fibrosing interstitial pneumonia of unknown cause, and its histopathologic changes often affect the subpleural and paraseptal parenchyma most severely. ${ }^{23}$ Both COPD and ILD are characterized by dyspnea and poor pulmonary function. The tumors are located in segmental and/or lobar bronchus in patients with CT-ADC, which may worsen the ventilation and exacerbate respiratory symptoms and airflow limitation of COPD and ILD.

The response rate to chemotherapy with or without radiotherapy was similar between patients with $\mathrm{CT}$-ADC and PT-ADC, suggesting that it is not necessary to change the treatment selection. The EGFR mutation rate of patients with pulmonary ADC was $51.6 \%$ in our study and the result was similar with that of PIONEER study. ${ }^{30}$ It is intriguing that though there was no statistical difference, patients with CT-ADC seemed to have lower EGFR mutation rate than those with PT-ADC. Together with the result that EGFR status was an independent prognostic factor in our study, the lower EGFR mutation rate of patients with CT-ADC may be another important reason for their poor prognosis. A possible explanation is that patients with EGFR mutation can benefit more from first- or second-line EGFR-TKI. ${ }^{31,32}$ But in our study, there were only $19 \%$ patients with EGFR mutation receiving EGFR-TKI as the first-line therapy and the subsequent therapy for other patients was unknown. In the future study, we will focus on patients treated with EGFR-TKI as first- or second-line therapy to investigate the prognosis of patients with CT-ADC and PT-ADC. Besides, EGFR plays a critical role in the pathogenesis and progression of multiple carcinomas including NSCLC. ${ }^{33,34}$ And EGFR mutant mice displayed defective lung branching morphogenesis and deficient alveolization and septation. ${ }^{34}$ The tendency of different EGFR mutation rates suggests that CT-ADC and PT-ADC may have different oncogenic mechanisms. The relationship between EGFR mutation and variant tumor locations should be confirmed by further studies.

Our study has several limitations. First, this was a retrospective study and we only included a small cohort. A prospective study and bigger cohort are desirable to further compare central and peripheral pulmonary ADC. Second, the tumor location was determined by radiological and 
bronchoscopic findings. It is possible that the pathological location is different. We excluded patients with an uncertain tumor location from our study to minimize such errors.

\section{Conclusion}

This is the first report to compare EGFR status, incidences of ILD and COPD, and treatment with and response between CT-ADC and PT-ADC. Tumor location plays a critical role in predicting prognosis and choosing therapeutic strategies. CTADC presents with more malignant clinical characteristics and poorer survival than PT-ADC. The lower rate of surgical resection in patients with $\mathrm{CT}-\mathrm{ADC}$ is an important reason for their poorer prognosis, and elevated rate of surgery might improve the survival of CT-ADC patients. Coexistence of CT-ADC and ILD/COPD is associated with poorer prognosis, and patients with such condition might need more care.

\section{Acknowledgments}

This work was supported by the grants from National Health and Family Planning Commission of China (201402011) and Shandong Key Research and Development Plan (2016CYJS01A03).

\section{Disclosure}

The authors report no conflicts of interest in this work.

\section{References}

1. Perez-Moreno P, Brambilla E, Thomas R, Soria J-C. Squamous cell carcinoma of the lung: molecular subtypes and therapeutic opportunities. Clin Cancer Res. 2012;18(9):2443-2451.

2. Kong J, Xu F, He M, Chen K, Qian B. The incidence of lung cancer by histological type: a population-based study in Tianjin, China during 1981-2005. Respirology. 2014;19(8):1222-1228.

3. Cheng I, Le GM, Noone A-M, et al. Lung cancer incidence trends by histology type among Asian American, native Hawaiian, and Pacific Islander populations in the United States, 1990-2010. Cancer Epidemiol Biomarkers Prev. 2014;23(11):2250-2265.

4. Devesa SS, Bray F, Vizcaino AP, Parkin DM. International lung cancer trends by histologic type: male:female differences diminishing and adenocarcinoma rates rising. Int $J$ Cancer. 2005;117(2): 294-299.

5. Sun W, Yang X, Liu Y, Yuan Y, Lin D. Primary tumor location is a useful predictor for lymph node metastasis and prognosis in lung adenocarcinoma. Clin Lung Cancer. 2017;18(1):e49-e55.

6. Ketchedjian A, Daly BDT, Fernando HC, et al. Location as an important predictor of lymph node involvement for pulmonary adenocarcinoma. J Thorac Cardiovasc Surg. 2006;132(3):544-548.

7. Koo HJ, Xu H, Choi C-M, et al. Preoperative CT predicting recurrence of surgically resected adenocarcinoma of the lung. Medicine. 2016;95(2): 2513.

8. Ito M, Yamashita Y, Miyata Y, et al. Prognostic impact of the primary tumor location based on the hilar structures in non-small cell lung cancer with mediastinal lymph node metastasis. Lung Cancer. 2012;76(1):93-97.
9. Gao Y-H, Guan W-J, Liu Q, et al. Impact of COPD and emphysema on survival of patients with lung cancer: a meta-analysis of observational studies. Respirology. 2016;21(2):269-279.

10. de Torres JP, Marín JM, Casanova C, et al. Lung cancer in patients with chronic obstructive pulmonary disease-incidence and predicting factors. Am J Respir Crit Care Med. 2011;184(8):913-919.

11. Gullón JA, Suárez I, Medina A, Rubinos G, Fernández R, González I. Role of emphysema and airway obstruction in prognosis of lung cancer. Lung Cancer. 2011;71(2):182-185.

12. Nishino M, Cardarella S, Dahlberg SE, et al. Interstitial lung abnormalities in treatment-naïve advanced non-small-cell lung cancer patients are associated with shorter survival. Eur J Radiol. 2015;84(5):998-1004.

13. Kanaji N, Tadokoro A, Kita N, et al. Impact of idiopathic pulmonary fibrosis on advanced non-small cell lung cancer survival. J Cancer Res Clin Oncol. 2016;142(8):1855-1865.

14. Kinoshita T, Azuma K, Sasada T, et al. Chemotherapy for non-small cell lung cancer complicated by idiopathic interstitial pneumonia. Oncol Lett. 2012;4(3):477-482.

15. Hohberger LA, Schroeder DR, Bartholmai BJ, et al. Correlation of regional emphysema and lung cancer: a lung tissue research consortiumbased study. J Thorac Oncol. 2014;9(5):639-645.

16. Lee T, Park JY, Lee HY, et al. Lung cancer in patients with idiopathic pulmonary fibrosis: clinical characteristics and impact on survival. Respir Med. 2014;108(10):1549-1555.

17. Mizushima Y, Kobayashi M. Clinical characteristics of synchronous multiple lung cancer associated with idiopathic pulmonary fibrosis. A review of Japanese cases. Chest. 1995;108(5):1272-1277.

18. Nagai A, Chiyotani A, Nakadate T, Konno K. Lung cancer in patients with idiopathic pulmonary fibrosis. Tohoku J Exp Med. 1992;167(3):231-237.

19. Lim J, Shin KM, Lee K, et al. Relationship between emphysema severity and the location of lung cancer in patients with chronic obstructive lung disease. AJR Am J Roentgenol. 2015;205(3):540-545.

20. Sakurai H, Asamura H, Watanabe S-Ichi, Suzuki K, Tsuchiya R. Clinicopathologic features of peripheral squamous cell carcinoma of the lung. Ann Thorac Surg. 2004;78(1):222-227.

21. Kanaji N, Sakai K, Ueda Y, et al. Peripheral-type small cell lung cancer is associated with better survival and higher frequency of interstitial lung disease. Lung Cancer. 2017;108:126-133.

22. Vogelmeier CF, Criner GJ, Martinez FJ, et al. Global strategy for the diagnosis, management and prevention of chronic obstructive lung disease 2017 report. Respirology. 2017;22(3):575-601.

23. Raghu G, Collard HR, Egan JJ, et al. An official ATS/ERS/JRS/ALAT statement: idiopathic pulmonary fibrosis: evidence-based guidelines for diagnosis and management. Am J Respir Crit Care Med. 2011;183(6):788-824.

24. Decaluwé H, Stanzi A, Dooms C, et al. Central tumour location should be considered when comparing N1 upstaging between thoracoscopic and open surgery for clinical stage I non-small-cell lung cancer. Eur J Cardiothorac Surg. 2016;50(1):110-117.

25. Davoli F, Bertolaccini L, Pardolesi A, Solli P. Video-assisted thoracoscopic surgery bronchial sleeve lobectomy. J Vis Surg. 2017;3:41.

26. Xu X, Chen H, Yin W, et al. Thoracoscopic half carina resection and bronchial sleeve resection for central lung cancer. Surg Innov. 2014;21(5):481-486.

27. Margaritopoulos GA, Vasarmidi E, Jacob J, Wells AU, Antoniou KM. Smoking and interstitial lung diseases. Eur Respir Rev. 2015;24(137):428-435.

28. Postma DS, Bush A, van den Berge M. Risk factors and early origins of chronic obstructive pulmonary disease. Lancet. 2015;385(9971):899-909.

29. Kenfield SA, Wei EK, Stampfer MJ, Rosner BA, Colditz GA. Comparison of aspects of smoking among the four histological types of lung cancer. Tob Control. 2008;17(3):198-204.

30. Shi Y, Au JS-K, Thongprasert S, et al. A prospective, molecular epidemiology study of EGFR mutations in Asian patients with advanced non-small-cell lung cancer of adenocarcinoma histology (pioneer). J Thorac Oncol. 2014;9(2):154-162. 
31. MokTS, WuY-L, Thongprasert S, et al. Gefitinib or carboplatin-paclitaxel in pulmonary adenocarcinoma. N Engl J Med. 2009;361(10):947-957.

32. Mok T, Yang J-J, Lam K-C. Treating patients with EGFR-sensitizing mutations: first line or second line-is there a difference? J Clin Oncol. 2013;31(8):1081-1088.
33. Normanno N, de Luca A, Bianco C, et al. Epidermal growth factor receptor (EGFR) signaling in cancer. Gene. 2006;366(1):2-16.

34. Sibilia M, Kroismayr R, Lichtenberger BM, Natarajan A, Hecking M, Holcmann M. The epidermal growth factor receptor: from development to tumorigenesis. Differentiation. 2007;75(9):770-787.

\section{Publish your work in this journal}

Cancer Management and Research is an international, peer-reviewed open access journal focusing on cancer research and the optimal use of preventative and integrated treatment interventions to achieve improved outcomes, enhanced survival and quality of life for the cancer patient. The manuscript management system is completely online and includes a very quick and fair peer-review system, which is all easy to use. Visit http://www.dovepress.com/testimonials.php to read real quotes from published authors. 